\title{
Gastric adenocarcinoma with enteroblastic differentiation: an unexpected cause of upper gastrointestinal bleeding
}

\author{
E. Dias ${ }^{1}$, J. Santos-Antunes ${ }^{1}$, A. C. Nunes ${ }^{1}$, J. A. Rodrigues ${ }^{2}$, J. Pinheiro², G. Macedo ${ }^{1}$ \\ (1) Gastroenterology Department, Centro Hospitalar de São João, Porto, Portugal; (2) Pathology Department, Centro Hospitalar de São João, Porto, Portugal.
}

\section{To the Editor,}

A 78-year-old male with previous medical history of hypertension, dyslipidemia, benign prostatic hyperplasia and colectomy for colon adenocarcinoma 16 years earlier presented to emergency department with melena for approximately 2 weeks. He denied hematemesis or hematochezia. He also denied other symptoms including abdominal pain, nausea, vomiting, fever, anorexia or weight loss. Usual medications included silodosin, simvastatin, losartan, hydrochlorothiazide, pantoprazole and midazolam. He denied recent intake of iron supplements or non-steroidal anti-inflammatory drugs. Physical examination was unremarkable except for pale skin. Laboratory studies revealed the presence of anemia (hemoglobin level: $7.1 \mathrm{~g} / \mathrm{dL}$ ). Leukocyte and platelet counts, liver tests, renal function, electrolyte levels, C-reactive protein and coagulation studies were all normal. Upper digestive endoscopy revealed red blood and blood clots in gastric lumen and a polypoid lesion with a diameter of approximately $20 \mathrm{~mm}$ located at the greater curvature of the proximal body with active oozing hemorrhage (Figure 1). Bleeding was successfully controlled with injection of diluted epinephrine at the base of the polyp and the patient was admitted in intermediate care unit for close monitoring.

During the following days, the patient maintained melena and required three units of packed red blood cells. Therefore, upper digestive endoscopy was repeated. There was still active oozing hemorrhage originating from the gastric polyp. Injection of diluted epinephrine followed by polypectomy with hot snare was performed. After this, the patient evolved favorably with no recurrent bleeding and stabilization of hemoglobin levels.

Histopathological examination of the resected polyp revealed an invasive adenocarcinoma with tubularpapillary architecture (Figure 2A), composed predominantly by neoplastic cells with clear glycogen-rich cytoplasm and irregular hyperchromatic nuclei (Figure 2B). Immunohistochemical analysis revealed diffuse nuclear expression of SALL4 (Figure 2C) and focal expression of glipican-3 (Figure 2D) and alpha-fetoprotein (Figure 2E). These features were consistent with gastric adenocarcinoma with enteroblastic differentiation. Computerized tomography scan of thorax, abdomen and pelvis did not reveal secondary involvement of lymph nodes, lungs, liver or other organs. Serum alpha-

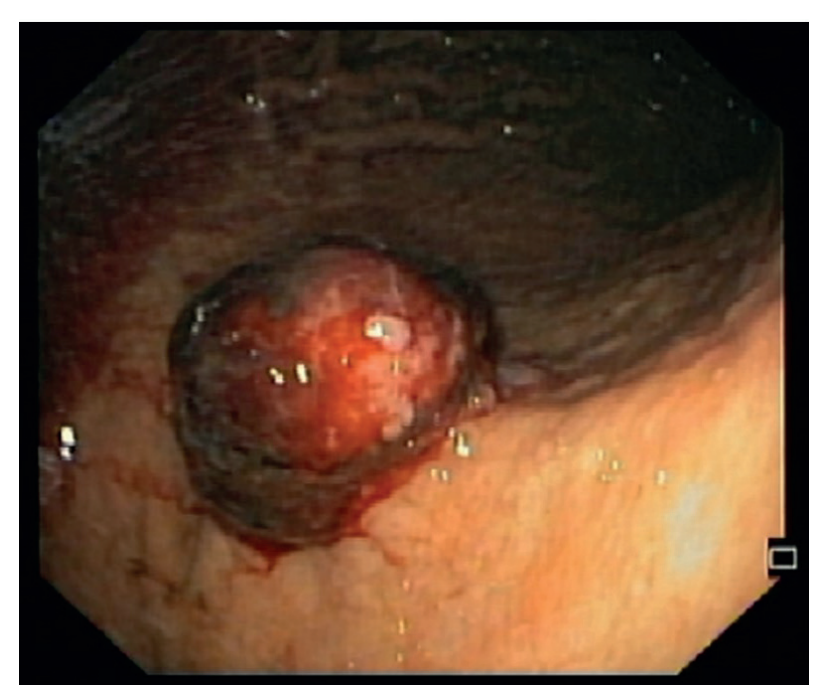

Figure 1. - Upper digestive endoscopy revealed a large polyp with a diameter of approximately $20 \mathrm{~mm}$ located at the greater curvature of the proximal body with active oozing hemorrhage.

fetoprotein level was normal. The patient undergone total gastrectomy with lymphadenectomy. After 2 months he is asymptomatic and there is no evidence of recurrence.

Gastric adenocarcinoma with enteroblastic differentiation is a rare malignancy, histologically characterized by the presence of a primitive intestine-like structure composed of cuboidal or columnar cells with clear cytoplasm, that stain positive for oncofetal proteins including glypican-3, SALL4 or alpha-fetoprotein (1). These malignancies appear to be associated with poor prognosis, with higher rates of lymphatic and venous invasion, lymph node metastasis and liver metastasis than conventional gastric cancer (2).

We report a case of gastric adenocarcinoma with enteroblastic differentiation presenting with melena and anemia requiring administration of packed red blood cells. Interestingly, in a retrospective clinicopathologic study involving 10 patients, half had symptoms related to upper gastrointestinal bleeding (one with melena, one

Correspondence to: Emanuel Dias, Gastrenterology Department, Centro Hospitalar de São João, Alameda Professor Hernâni Monteiro, 4200-319, Porto, Portugal.

E-mail: ea.dias91@gmail.com

Submission date : 15/05/2021

Acceptance date : 18/07/2021 


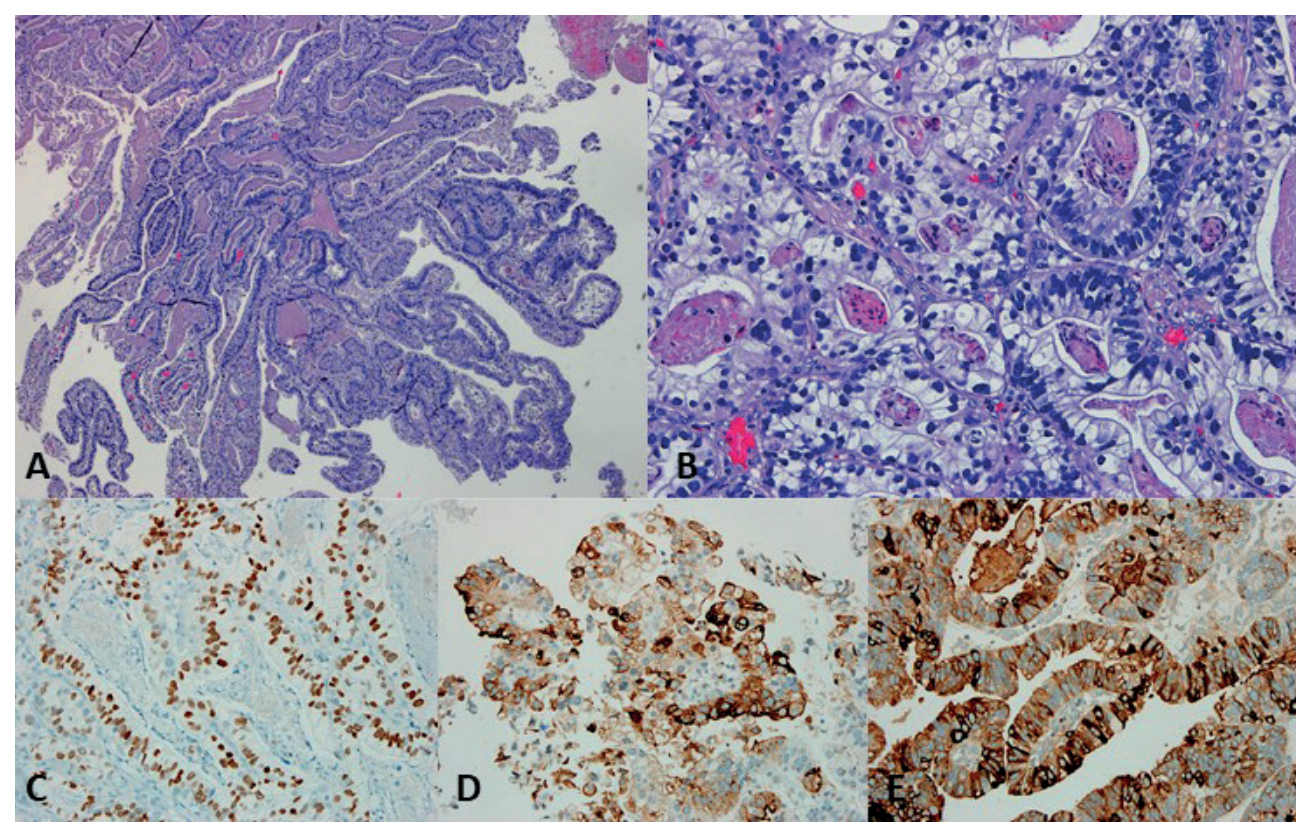

Figure 2. - A. Adenocarcinoma with tubular papillary architecture (H\&E, 40x). B. Neoplastic glands composed of cells with abundant clear cytoplasm, reminiscent of fetal gut (H\&E, 200x). C-E. Immunohistochemical findings in enteroblastic adenocarcinoma. Expression of: C. SALL4. D. glipican-3. E. alpha-fetoprotein (IHC, 200x).

with hematemesis and three with iron-deficiency anemia) (3). Therefore, gastric adenocarcinoma with enteroblastic differentiation is a rare but possible cause of upper gastrointestinal bleeding and this case demonstrates that, although unusual, it may be severe enough to cause anemia requiring blood transfusion.

Keywords: gastric adenocarcinoma, gastric adenocarcinoma with enteroblastic differentiation, gastrointestinal bleeding, melena.

\section{Financial support}

The authors received no financial support for the research, authorship, and/or publication of this article.

\section{Conflict of interest}

No potential conflict of interest relevant to this article was reported.

\section{References}

1. YAMAZAWA S., USHIKU T., SHINOZAKI-USHIKU A., HAYASHI A., IWASAKI A., ABE H., et al. Gastric Cancer With Primitive Enterocyte Phenotype: An Aggressive Subgroup of Intestinal-type Adenocarcinoma. Am J Surg Pathol. 2017; 41(7): 989-97.

2. MURAKAMI T., YAO T., MITOMI H., MORIMOTO T., UEYAMA H., MATSUMOTO K., et al. Clinicopathologic and immunohistochemical characteristics of gastric adenocarcinoma with enteroblastic differentiation: a study of 29 cases. Gastric Cancer. 2016; 19(2): 498-507.

3. KURODA N., YORITA K. Clinicopathologic study of 10 cases of gastric adenocarcinoma with hepatoid or enteroblastic differentiation. Pol J Pathol. 2018; 69(2): 128-35. 